

\title{
Ports militaires, les arsenaux passent le relais aux universités
}

Raymonde Séchet

\section{To cite this version:}

Raymonde Séchet. Ports militaires, les arsenaux passent le relais aux universités. Les Annales de la Recherche Urbaine, 1992, 55-56, pp.88-96. halshs-01095659

\section{HAL Id: halshs-01095659 \\ https://shs.hal.science/halshs-01095659}

Submitted on 29 Nov 2016

HAL is a multi-disciplinary open access archive for the deposit and dissemination of scientific research documents, whether they are published or not. The documents may come from teaching and research institutions in France or abroad, or from public or private research centers.
L'archive ouverte pluridisciplinaire HAL, est destinée au dépôt et à la diffusion de documents scientifiques de niveau recherche, publiés ou non, émanant des établissements d'enseignement et de recherche français ou étrangers, des laboratoires publics ou privés. 


\section{Ports militaires, les arsenaux passent le relais aux universités} Raymonde Séchet

\section{Citer ce document / Cite this document :}

Séchet Raymonde. Ports militaires, les arsenaux passent le relais aux universités. In: Les Annales de la recherche urbaine, N55-56, 1992. Grandes villes et ports de mer. pp. 89-96;

http://www.persee.fr/doc/aru_0180-930x_1992_num_55_1_1677

Document généré le 03/10/2016 


\section{Résumé}

La crise économique des ports militaires s'aggrave du fait du désarmement international. Pour ouvrir à la population des perspectives de promotion, l'Etat et les collectivités locales développent ou créent des universités. Mais les villes se retrouvent en concurrence pour les formations les plus spécialisées tout en dédaignant les filières générales, non articulées à l'économie régionale.

\section{Abstract}

Raymonde Séchet, Military ports.

The universities are taking over from the naval dockyards

The economic woes of military ports have increased as a result of international disarmement. To open up new prospects for advancement to people, the government and local authorities are creating and expanding universities. But cities are competing for highly specialised study programs and disregarding broader ones that are less relevant to the region's economy.

\section{Zusammenfassung}

Raymonde Séchet, Militärhäfen -die Waffenkammern weichen den Universitäten

Die internationale Abrüstung, verstärkt die Wirtschaftskrise in den Militärhäfen. Um der Bevölkerung Aufstiegchancen zu eröffnen, entwickeln oder gründen Staat und regionale Körperschaften Universitäten. Aber die Städte konkurrieren untereinander um hochspezialisierte Ausbildungsgänge und weigern sich, weniger spezielle und der regionalen Wirtschaftstruktur weniger verbundene Studienfächer anzubieten.

\section{Resumen}

Raymonde Séchet, Puertos militares, las universidades reemplazan a los arsenates

La crisis económica de los puertos militares se agrava debido al desarme internacional. Para ofrecer perspectivas de promoción a sus poblaciones, el Estado y las entidades locales desarrollan o crean universidades. Pero las ciudades insisten en dar formaciones especializadas y desdeñan las ramas generates, no articuladas a la economía general. 


\section{PoRTS MILITAIRES, LES ARSENAUX PASSENT LE RELAIS AUX UNIVERSITÉS}

Raymonde Séchet

\section{Le ciel est chagrin au-dessus de Cherbourg. Et assez nuageux au-dessus de Brest et de Lorient.' \\ $V$ \\ ictimes de l'évolution des rela-} tions internationales et des technologies militaires, des villes comme Toulon, Brest, Cherbourg, et à un moindre degré Lorient, dont l'urbanisme, la puissance, la culture se sont structurés autour d'un arsenal et d'un port militaire, subissent actuellement les effets des lois de programmation militaire et de la réduction des budgets de la Défense. Les répercussions économiques, sociales, urbaines sont telles que ces villes sont en pleine période de recomposition identitaire. La consolidation de l'appareil de formation, et notamment la mise en place ou l'étoffement de la fonction universitaire, apparaissent comme un des éléments majeurs de cette entreprise de redynamisation urbaine. Ces quatre ports sont restés à l'écart de la fonction universitaire jusqu'aux années soixante pour Brest et Toulon, jusqu'à aujourd'hui pour Cherbourg et Lorient. Ce retard, également sensible dans d'autres villes portuaires comme Dunkerque, Le Havre, Saint-Nazaire, a pu constituer un handicap. Aussi, ces villes ont été parmi les plus sensibles aux possibilités de développement universitaire, notamment dans le cadre du plan Université 2000. Cependant la mobilisation pour les doter des équipements de formation dignes de leur place dans le réseau urbain français ne va pas sans ambiguités, sans contradictions, sans limites.

\section{L'affaiblissement de la tutelle de l'Etat}

L'histoire de ces villes qui se confond avec celle de leur port militaire et de leur arsenal est aussi celle de la marine royale puis nationale. Depuis leur création, leur vie a été rythmée par une alternance de temps prospères, en période de guerre et de tension internationale, et de phases de stagnation ou de déclin lors des intermèdes de paix. Les effectifs employés dans les arsenaux ont connu une évolution cyclique et sont actuellement en phase de réduction. Plusieurs études laissent supposer que de 1992 à 1995, près de 100000 emplois sur les 300000 que compte l'industrie française de l'armement seraient menacés².
Au cours des trois dernières décennies, ces villes ont toutes, plus ou moins, été confrontées aux mêmes difficultés et à la même nécessité d'une conversion économique et sociale. Les problèmes sont souvent apparus au grand jour lorsque la phase de reconstruction s'est achevée pour celles que leurs positions stratégiques et leurs fonctions avaient particulièrement exposées aux bombardements. Ainsi, à Brest «Un seul monument public survécut : l'église de Recouvrance. Les travaux commencèrent aussitôt et durèrent quatorze ans... Un instant

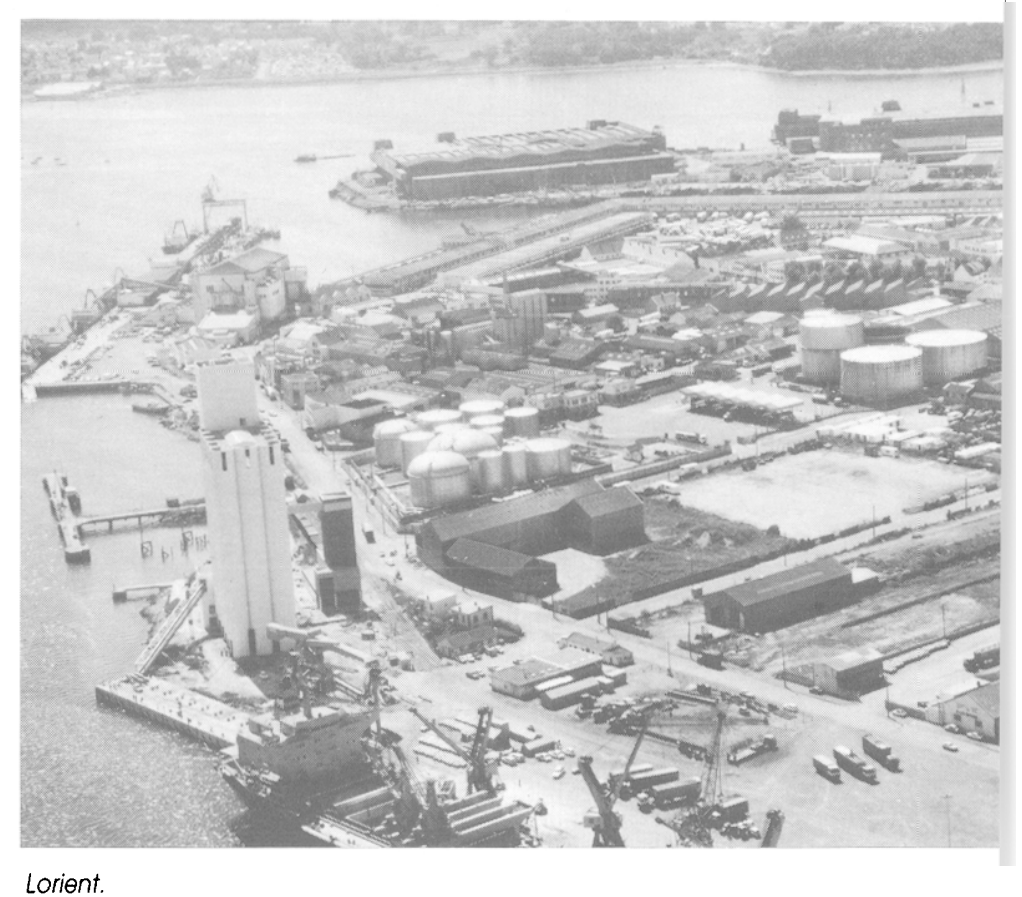

dramatique surgit en 1958. Brest pendant quatorze ans avait vécu de sa propre reconstruction. Pour elle avait travaillé tout l'ouest du Léon, pour elle les carrières et les sablières avaient fonctionné à plein, pour elle l'émigration lointaine s'était stoppée. Qu'allaient devenir

\footnotetext{
1. François Simon, Ouest-France, 2-3 mai 1992.

2. cf. Le Monde, 30 juin 1992, p. 40.

I.es Amales de la Recherche Urhaine n $155-56.0180-930-1 X .92 / 56 / 89: 8$ @ MELATT
} 
tant d'emplois et tant d'entreprises, une fois les Brestois relogés? »3.

Un processus de diversification des activités s'est alors amorcé. A Brest comme ailleurs en Bretagne, les industries électriques, électroniques, agro-alimentaires et de l'habillement ont occupé les nouvelles zones industrielles pour employer une main-d'oeuvre peu qualifiée. Au cours de cette phase. la ville a semblé rompre avec le déterminisme économique généré par sa position maritime pour connaitre une évolution plus proche de celle du contexte régional breton. A Cherbourg et dans le NordCotentin, la politique de décentralisation opérée dans le cadre de l'aménagement du territoire a favorisé la venue

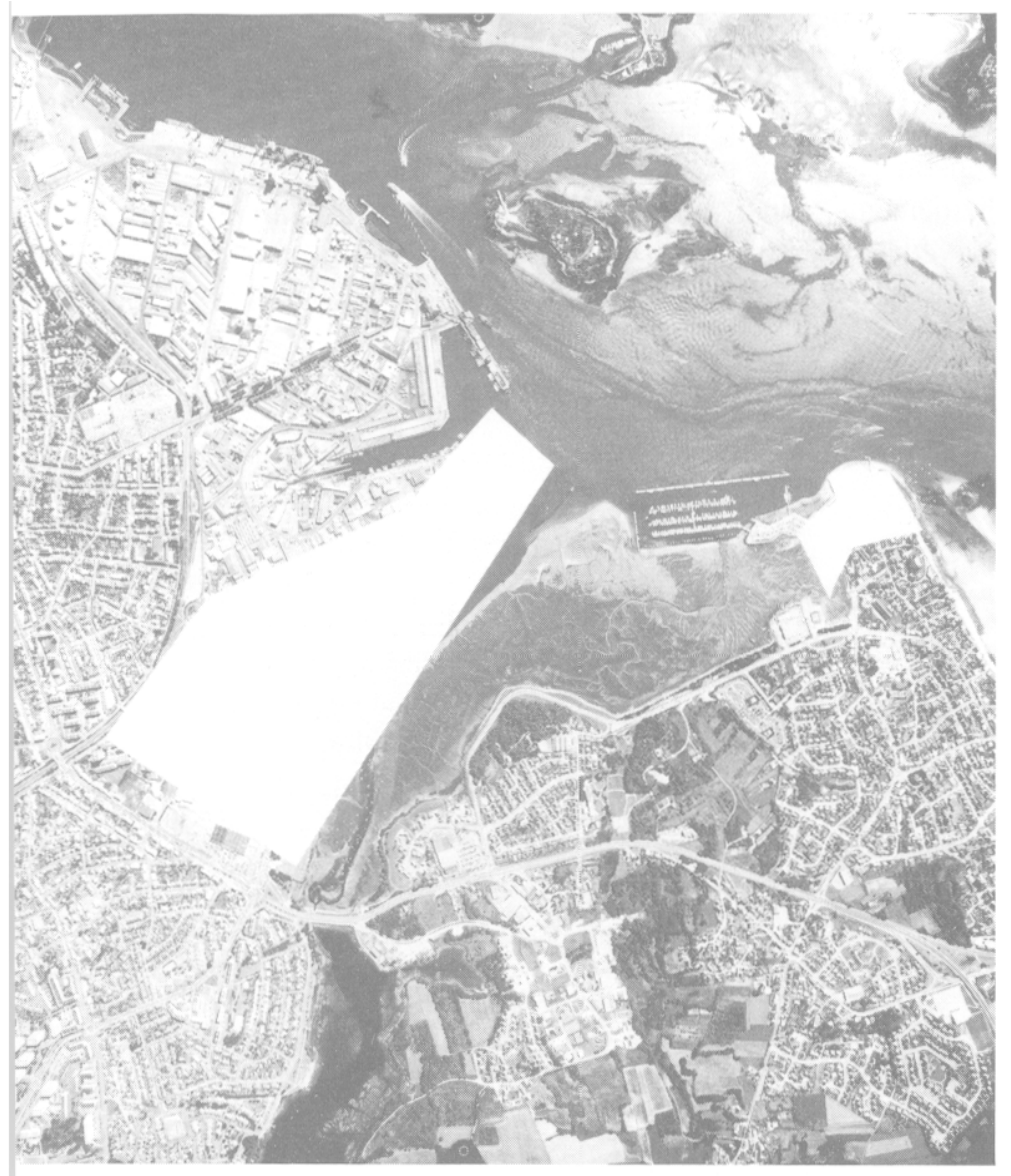

Rade de Lorient, en blanc : la base de sous-marins.

de CIT-Alcatel et de Thomson et le développement de l'habillement, ouvrant à la main-d'œuvre féminine un tissu industriel dominé jusqu alors par la DCAN (Direction des Constructions et Armes Navales). Cependant le développement d'une autre activité d'Etat, le nucléaire, avec l'usine de retraitement de La Hague et la centrale nucléaire de Flamenville, a ici maintenu, si ce n'est renforcé, la position dominante de l'Etat dans la vie économique du bassin d'emploi. D'autant que la construction navale civile n'a ici de privé que les capitaux, car la production a traditionnellement été essentiellement militaire (Construction Mécanique de Normandie-Félix Amiot).
Par ailleurs, le bassin d'emploi du Nord-Cotentin a été particulièrement affecté par les restructurations en cours à l'échelon national (et international) dans la construction navale, la téléphonie, l'habillement. Après l'effetdopage produit par les grands chantiers du nucléaire, sont venus les «lendemains de l'optimisme» ${ }^{+}$. Au total le poids économique de l'Etat reste fort dans ces villes, moins à Lorient riche de son port de pêche et de son arrière-pays agro-alimentaire, tellement à Cherbourg que le 2 juillet 1992, le Secrétaire d'Etat à l'aménagement du territoire, André Laignel, est venu dire aux Cherbourgeois que leur ville «ne pourra éternellement dépendre à plus de $80 \%$ de la commande publique».

L'amorce de diversification n'en constitue pas moins une mutation du système de relations à l'espace. Mal insérées dans le réseau urbain français, Brest, Toulon, Cherbourg, Lorient présentent un décalage entre leur position dans le maillage administratif du pays (Cherbourg, Brest, Lorient ne sont que sous-préfectures et Toulon n'a supplanté Draguignan comme préfecture du Var qu'en 1975) et leur surface économique et démographique : Cherbourg compte 92045 habitants, Brest 201 480, Lorient 115488 , Toulon 437553 habitants, ce qui place ces villes aux soixante-cinquième, vingt-neuvième, cinquante et unième, neuvième rang de la hiérarchie urbaine française'. Elles ne doivent leur importance ni à une position de carrefour ni à un phénomène de polarisation, ni même à un gisement local au sens classique du terme mais à la volonté de pouvoirs politiques, qu'ils aient été monarchique, d'empire ou républicain, de disposer de flottes de guerre puissantes et prêts pour cela à exploiter les quelques sites côtiers les plus favorables.

La nature de l'activité dominante a généré des identités locales particulièrement marquées, dont témoigne la mobilisation exceptionnelle de la population de Cherbourg et du Cotentin à l'occasion des manifestations du ler mai après l'annonce par le ministre de la Défense au printemps 1992 du départ de la flotille du nord (450 marins) et de la suppression de 240 emplois à l'arsenal. Ces unités urbaines à l'économie tournée vers la mer, et qui ont toujours entretenu des relations privilégiées mais de dépendance au pouvoir central, étaient faiblement ancrées dans leur espace régional. Est-il choquant de dire que Cherbourg, Brest, Lorient ont été plus proches de Paris que de Saint-Lô, Quimper ou Vannes alors que. jusqu'à la Seconde guerre mondiale, elles ont fonctionné comme des principautés insulaires? Leurs environs ont surtout représenté les réservoirs de main-d'œuvre nécessaires à tout gonflement des effectifs des arsenaux.

3. André Meynier, Atlas et géographie de la France moderne : la Bretagne, p. 89. 4. Buléon P., "Nord-Cotentin : les lendemains de l'optimisme», Caen. Bulletin di Centre d'Etudes Régionales et d'Aménagement 27, Caen. Septembre 1985.

5. RGP 1990. Population des unités urbaines selon la définition de 1990. A titre de comparaison, les agglomérations de Saint-lô, Quimper, Vannes comptent $26577,65954,45644$ habitants. 
L'insertion régionale amorcée par la diversification économique passe actuellement par l'adaptation des ports à de nouveaux trafics ${ }^{6}$ et surtout par la recherche d'une fonction de métropole les dotant d'une réelle influence régionale, et donc par le développement d'équipements et de services leur permettant de s'affirmer en tant que villes, et de rompre avec leur image de ports industriels et guerriers, de cités de marins et d'ouvriers.

\section{L'université}

\section{pour une nouvelle dynamique}

La création d'universités et d'établissements d'enseignement supérieur témoigne le mieux de l'aspiration à un nouveau développement. Déjà, dans les années soixante, la dotation en universités de tous les chefs-lieux académiques, préfectures de région, métropoles d'équilibre (Nantes) ainsi que d'autres agglomérations importantes (Brest, Le Mans, Le Havre) avait complété et resserré le maillage universitaire. Le mouvement connaît aujourd'hui une nouvelle vigueur avec la politique des antennes délocalisées et les projets de création d'universités. Les villes portuaires, et notamment les ports militaires, ont largement bénéficié de ces nouvelles implantations avec:

- des universités de création récente, Brest, Toulon... et Le Havre ;

- l'émergence de nouveaux sites universitaires : Lorient où les trois universités bretonnes sont présentes à travers des formations délocalisées, promue, avec Vannes, pôle de développement universitaire dans la perspective de la création d'une quatrième université bretonne ; Cherbourg où les premières formations délocalisées de Caen sont entrées en activité en 1991... mais aussi Dunkerque et La Rochelle soit deux des trois nouvelles universités créées en province dans le cadre du plan Université 2000 (la troisième étant prévue à Arras).

Actuellement les effectifs restent modestes et aucune de ces villes ne peut être considérée comme ville universitaire selon le critère rapport étudiants/population de l'agglomération. Pour le mieux, à Brest en 1990, l'université comptait 11372 étudiants, soit un étudiant pour dix-huit habitants ${ }^{7}$. Aussi ces collectivités territoriales se sont-elles largement impliquées dans les débats suscités récemment par les mutations profondes de l'université et par le renforcement de la carte universitaire. Les universités y sont perçues comme un enjeu majeur économique, social, politique du développement local et comme une des clés du changement d'image. Le développement universitaire est donc intégré dans les projets d'agglomération : "Dans la mesure où l'enseignement supérieur et la recherche constituent un des moteurs d'une dynamique urbaine, il est apparu essentiel que la réflexion sur le redéploiement universitaire soit associée à la démarche de consolidation de l'économie locale, de reconquête de la ville par l'aménagement, et de séduc- tion» ${ }^{8}$. En conformité avec cette option, la qualité des espaces et des bâtiments universitaires doit contribuer à faire de l'université un atout pour le développement de l'agglomération. Dans l'esprit des architectes, les

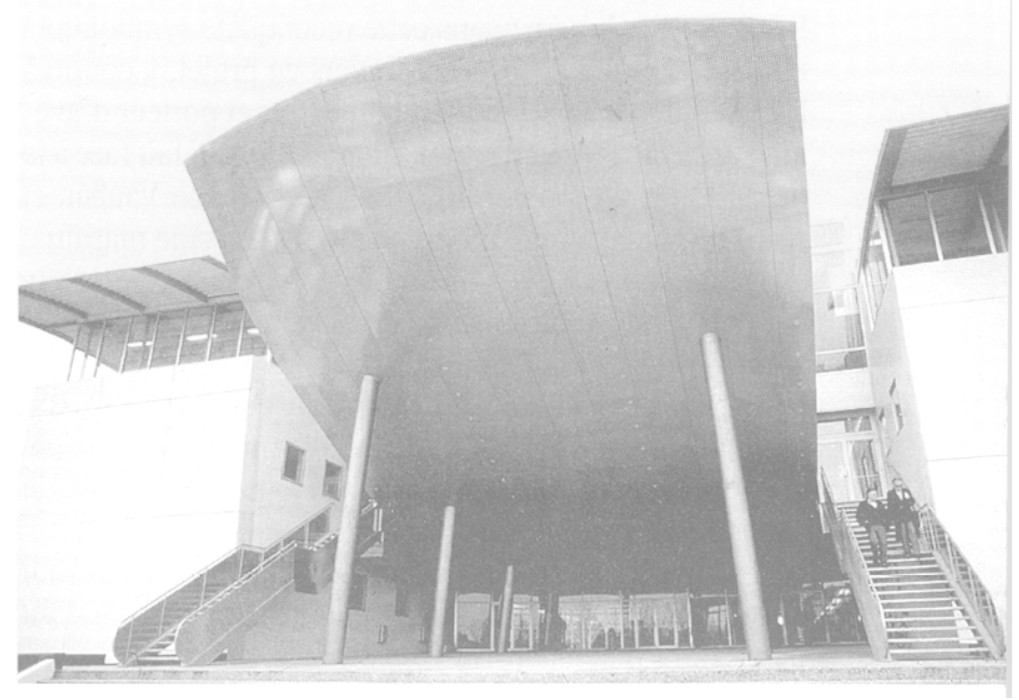

Bâtiments universitaires de Lorient.

aspects formels de l'architecture sont d'ailleurs au service de la symbolique urbaine, tout particulièrement lorsque l'histoire a anéanti le patrimoine architectural préexistant.

L'un des deux volets du projet d'agglomération brestois vise à une réarticulation entre le centre et les autres quartiers de la ville ; un centre-ville lui-même recomposé pour être à la hauteur des ambitions de l'agglomération (cf les fontaines de Martha Pan dans la Rue de Siam). Dans ce cadre prennent place les débats sur la localisation des nouveaux équipements universitaires : transfert de l'Ecole Nationale des Ingénieurs de Brest et de l'Institut des sciences de l'agriculture et du monde rural sur le technopôle de Brest-Iroise ; réaménagement du campus du Bouguen en position péricentrale ; surtout, choix d'un site universitaire en centre-ville fait à l'unanimité des partenaires à l'automne 1990 (disponibilités foncières du site Clémenceau-Foch).

6. On peut reprendre ici la remarque d'Alain Chauvet relative à l'ensemble des ports de la façade atlantique française : "Après avoir dû renoncer à concurrencer ceux de la Mer du Nord et de la Méditerranée sur leur propre terrain ( sidérurgie, pétrochimie) ils ont plus modestement développé quelques lignes nouvelles ef quelques trafics spécifiques (tourteaux, bois, ferrailles ...). L'un des objectifs essentiels de beaucoup de ces ports est d'intégrer davantage l'économie portuaire à celle de la région." Rapport de recherche à la DATAR La Façade atlantique : Stratégies et prospective de développement, septembre 1991.

7. Contre, à titre de comparaison, 32551 étudiants pour 245065 habitants dans l'agglomération rennaise soit un étudiant pour huit habitants. L'ensemble des effectifs post-bac était de 17352 étudiants à Brest et 42245 à Rennes.

8. Rapport «Brest : une ville universitaire pour la Bretagne occidentale» présenté par l'agence d'urbanisme de la communauté urbaine de Brest au colloque Les villes et l'explosion universitaire, Dunkerque, 1991 
L'autre volet du projet d'agglomération cherche à établir un nouveau rapport de la ville à la mer grâce à une reconquête symbolique du site, la Penfeld, le port, la rade, notamment par la recomposition ambitieuse du front de mer. Le projet de François Chochon pour un institut européen des sciences de la mer au port de commerce de Brest devait s'insérer dans cette reconquête symbolique. Pour lui, la ville de Brest, à travers ce projet, a confié à l'université «un rôle emblématique fort et porteur d'avenir, sur sa façade la plus importante, celle qui fait face à la mer». D'où un projet s'intégrant au système Vauban et réalisant «un cadrage monumental du paysage maritime avec, au lointain, la presqu'île de Crozon» ${ }^{9}$. Avec la même volonté de production de lieux métaphoriques, les architectes G. et D. Peneau, pour les locaux universitaires de Lorient qui vont ouvrir en octobre 1992, ont multiplié les références marines avec un bâtiment doté d'une proue (grand amphithéâtre), de hublots et de superstructures en forme de vagues.

Aucune ville un peu importante n'échappe aujourd'hui au désir technopolitain et universitaire qui lui permettrait de prendre place dans la concurrence entre villes européennes. L'essor des activités de formation continue, la diversification des relations d'échanges et de coopération entre la société locale et l'université supposent une insertion régionale accrue de l'université et des universitaires. Les collectivités territoriales attendent de l'université qu'elle forme des cadres et génère des emplois, qu'elle soit un facteur de dynamisme intellectuel et culturel, d'affirmation identitaire et de promotion d'une image de modernité. On attend du cadre relationnel international dans lequel s'opèrent les activités de recherche, et aussi de plus en plus l'enseignement, qu'il accroisse le rayonnement international des collectivités territoriales.

\section{L'ancrage sur le tissu régional}

En 1958, «Brest a joué deux cartes, la scolarisation et l'industrialisation... Compléter les institutions déjà vivaces, fournir de la pâture à l'appétit intellectuel des Finistériens, tel fut le but de la création d'un enseignement supérieur» ${ }^{10}$. En fait, Brest a été dans la situation qui est aujourd'hui celle des villes moyennes aspirant à accueillir des antennes délocalisées : «La création d'un pôle universitaire ne rencontre pas l'adhésion seulement pour des raisons d'utilité/coût ; elle permet aussi l'affirmation d'une certaine légitimité locale. La ville moyenne peut espérer jouir d'un rayonnement culturellement du même type que celui dont bénéficie la métropole régionale.... La délocalisation universitaire participe à la construction ou à la recomposition du territoire à travers sa fonction d'intégration sociale et locale, au même titre sans doute que d'autres politiques publiques locales, culturelles" par exemple. Elle est posée, affirmée par ses acteurs comme un élément du développement local... Elle est davantage que du développement local, elle est aussi d'un ordre symbolique.»12

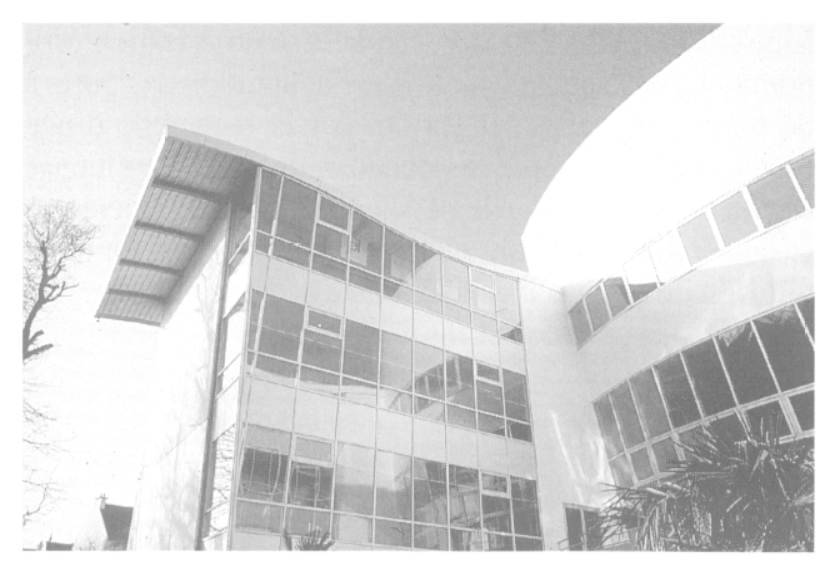

Bâtiments uriversitaires de Lorient.

L'Université a été perçue comme un moyen de susciter des dynamiques nouvelles tout en favorisant l'ancrage régional de Brest. La municipalité puis la communauté urbaine de Brest, voyant en Brest la ville universitaire de Bretagne occidentale, ont toujours eu une politique volontariste pour la création puis l'essor de l'UBO (Université de Bretagne Occidentale). Les perspectives actuelles de croissance des effectifs (de 12000 étudiants en 1989 à 21000 en 1994) (13 $^{13}$ ont donné «l'impulsion nécessaire à la saisie d'un enjeu à court terme consistant à faire reconnaître, non seulement à l'UBO la place à laquelle elle prétendait dans l'aménagement du territoire universitaire régional, mais aussi, à la ville, son rôle de métropole» ${ }^{1+}$... qui reste à prouver. L'objectif principal du plan de développement de l'université de Brest pour les années 1990-1993 est de diversifier les filières de formation, notamment par la multiplication des formations de deuxième et troisième cycles, afin de conforter la ville dans sa position de second pôle universitaire en Bretagne. La reconnaissance d'une université est supposée reposer sur la qualité de la recherche - et des filières d'enseignement qui en découlent — d'où la concentration des efforts sur quelques axes majeurs dont on souhaite localement qu'ils définissent des pôles d'excellence : «dans le domaine scientifique, quatre orientations sont privilégiées : les sciences de la mer, l'agro-alimentaire, l'électronique, la chimie fine et la chimie marine. Elles visent à se rapprocher des besoins de l'économie régionale et des préoccupations de certains organismes de recherche, tel IFREMER, pour développer un centre

9. F. Chochon dans MEN, Programme Architecture Nouvelle-Université 2000.

10. André Meynier, op. cité, p. 90.

11. Ainsi, le Quartz à l'architecture volontairement novatrice qui offre une salle de spectacles de 1500 places et des créations chorégraphiques et théâtrales de haut niveau est aujourd'hui une des bases de la politique de séduction de Brest.

12. Daniel Filatre, "Villes moyennes et universités, les enjeux des antennes universitaires》, Espaces et Sociétés n`59, 1990, p. 119-127.

13. Prévisions établies dans le cadre des assises locales Université 2000 ; le rapport régional se base sur un effectif prévisible de 18000 étudiants.

14. cf. note 8 . 
de recherche de rang international. Ainsi, l'université se doterait d'une identité et d'une image de marque qui serviraient aussi la ville.»15 Deux de ces spécialisations - l'agro-alimentaire et l'électronique — sont en relation avec le marché régional de l'emploi ; les deux autres réaffirment la dimension maritime de Brest, mais dans une relation à la mer modernisée, plus scientifique et moins guerrière. Ces choix que certains qualifieront de vocations ${ }^{16}$, permettent aujourd'hui à Brest de conforter ses prétentions régionales alors que la création en 1969 du centre de recherche bretonne et celtique a déjà contribué à l'affirmation de l'identité bretonne de l'UBO et de Brest.

Le cas cherbourgeois ${ }^{17}$ illustre également la préférence accordée à des établissements ouverts sur la production en fonction des spécificités économiques locales, à des formations liées au développement des technologies nouvelles et plus porteuses en termes de promotion urbaine. En plus des BTS offerts par les lycées cherbourgeois (chaudronnerie, maintenance, contrôle industriel et régulation automatique, bureautique trilingue), des établissements d'enseignement supérieur ont été créés : l'Institut des techniques de la mer, ouvert en 1988, offre 65 places à des étudiants recrutés nationalement ; en 1991, un département Gestion des Entreprises et des Administrations a été ouvert aux côtés des deux déjà en fonctionnement à l'IUT (Génie Electrique Informatique, Techniques de Commercialisation). A cette même rentrée 1991, Cherbourg a accueilli son premier cycle universitaire délocalisé : un Deug sciences physiques pour l'ingénieur pouvant former 60 étudiants par année, ce qui n'aura bien sûr aucun effet sur les effectifs caennais. La communauté urbaine de Cherbourg a soutenu deux autres projets en arguant toujours d'un ancrage sur le tissu économique local : d'une part, un Deug LEA Anglais/Allemand et/ou Japonais en fonction des échanges économiques et touristiques locaux ; de l'autre, un Deug Biologie qui permettrait de constituer avec Intechmer un pôle régional des techniques de la mer mais qui entre en concurrence avec la demande formulée par Saint-Lô dans la perspective d'un pôle agro-alimentaire. En fait, l'objectif principal des acteurs économiques et politiques locaux (Conseil Régional, Conseil Général, Communauté Urbaine, IUT, Chambre de commerce et d'industrie, Chambre syndicale des industriels métallurgistes de la Manche, établissements locaux des entreprises nationales comme COGEMA, DCAN, EDF) a été d'obtenir la création d'une école d'ingénieurs dans le créneau du génie industriel et offrant trois options (systèmes d'information industriels, systèmes de production automatisée, sytèmes de maintenance en milieux hostiles). Création annoncée pour 1993 par le Secrétaire d'Etat à l'aménagement du territoire le 2 juillet 1992.

Ainsi, à Brest comme à Cherbourg, le développement de la fonction universitaire, et notamment de formations spécialisées associées à technologie et modernisme pour donner une plus grande lisibilité aux universités et aux villes, constitue un élément majeur des politiques de promotion et de changement d'image des villes. Les aspirations de ces villes ouvrières et militaires sont de même nature que celles de Dunkerque : «L'image de la ville laborieuse, industrielle n'est porteuse de développement que si elle se croise avec celle de la qualité, de la technologie. Le projet universitaire n'amène pas à renverser l'image de la ville mais à la faire évoluer en la complé-tant: l'image scientifique s'ajoute à l'image productive et ne la remplace pas. Elle change le niveau de la ville» ${ }^{18}$. Par ses choix de formations universitaires de troisième cycle, Toulon semble plus fidèle à sa spécialisation économique : DESS matériaux en milieux marins et extrêmes, DESS défense et sécurité, DESU stratégie et défense.

\section{La spécialisation contre la polarisation?}

La spécialisation universitaire, telle qu'elle apparait souhaitée à Brest, à Cherbourg, à Lorient (secteur des matériaux composites justifié par la présence de la DCAN et de l'Institut de recherche des matériaux avancés), et ailleurs en France avec la multiplication de formations professionnalisées, est, en théorie, une manière $\mathrm{d}$ '«extraire en partie les universités des systèmes régionaux de zone d'influence urbaine pour en faire des éléments spécifiques de positionnement national et européen des sites supports»'19.

L'université demeure, par la liaison enseignement/ recherche, un lieu de production des savoirs fondamentaux préalables à toute innovation. La diffusion des établissements d'enseignement supérieur dans le réseau urbain devrait engendrer un développement dispersé des activités de recherche. Or, les activités de recherche sont moins dépendantes de la répartition de la population que de la qualité des sites et des avantages d'agglomération. Aussi, «la logique du développement de la recherche répond au principe de l'excellence, avec pour mesure la

\footnotetext{
15. cf. note 8 .

16. Notre nuance rhétorique peut renvoyer à Robert Ferras pour qui «il n'y a pas de prétendue vocation, terme qui traîne encore trop souvent dans les manuels de géographie urbaine, mais des réalités qui prennent en compte les conditions de la production à venir, selon l'attrait de la ville, la main-d'oeuvre que l'on peuty trouver, la paix sociale garantie ou le bon vouloir d'une municipalité... Nulle vocation n'est indépendante de l'histoire et de ceux qui la font, avec d'un côté un environnement physique, indéniable mais tout relatif, de l'autre un milieu social agissant mais daté. Et, à la charnière, une profusion d'images." Extrait de Ville, paraître, être à part, GIP RECLUS 1990, pages 131 et 135.
}

17. Etudié par Anne Briard et Marie-Laure Faudemer dans le cadre d'un DEA (Les antennes universitaires de Saint-lô et Cherbourg) soutenu à Caen en 1991

18. Citations extraites du rapport "Construction d'un projet d'université dans un projet de ville" présenté par l'agence d'urbanisme et de développement de la région Flandre-Dunkerque au colloque Les villes et l'explosion universitaire, Dunkerque, 1991.

19. Debarbieux B., "Quel aménagement du territoire pour l'université française?», l'Espoce géogrcphique 1991.3, p. 205. 
visibilité internationale $»^{20}$ et «sa logique de localisation est une logique d'activité productive de haute technologie, et non une logique de localisation de service banal à la population» 2 . Le ministre, H. Curien, distingue les organismes de recherche à vocation large, très largement répartis sur le territoire national et qui accompa- l'ancienneté des universités : les plus anciennes dispensent les formations les plus banales pour le plus grand nombre mais délivrent aussi la majorité des formations les plus rares. Ces universités, souvent implantées dans les grandes villes, peuvent répondre aux besoins de la fonction de métropole (diversité et rareté) autant qu'à la

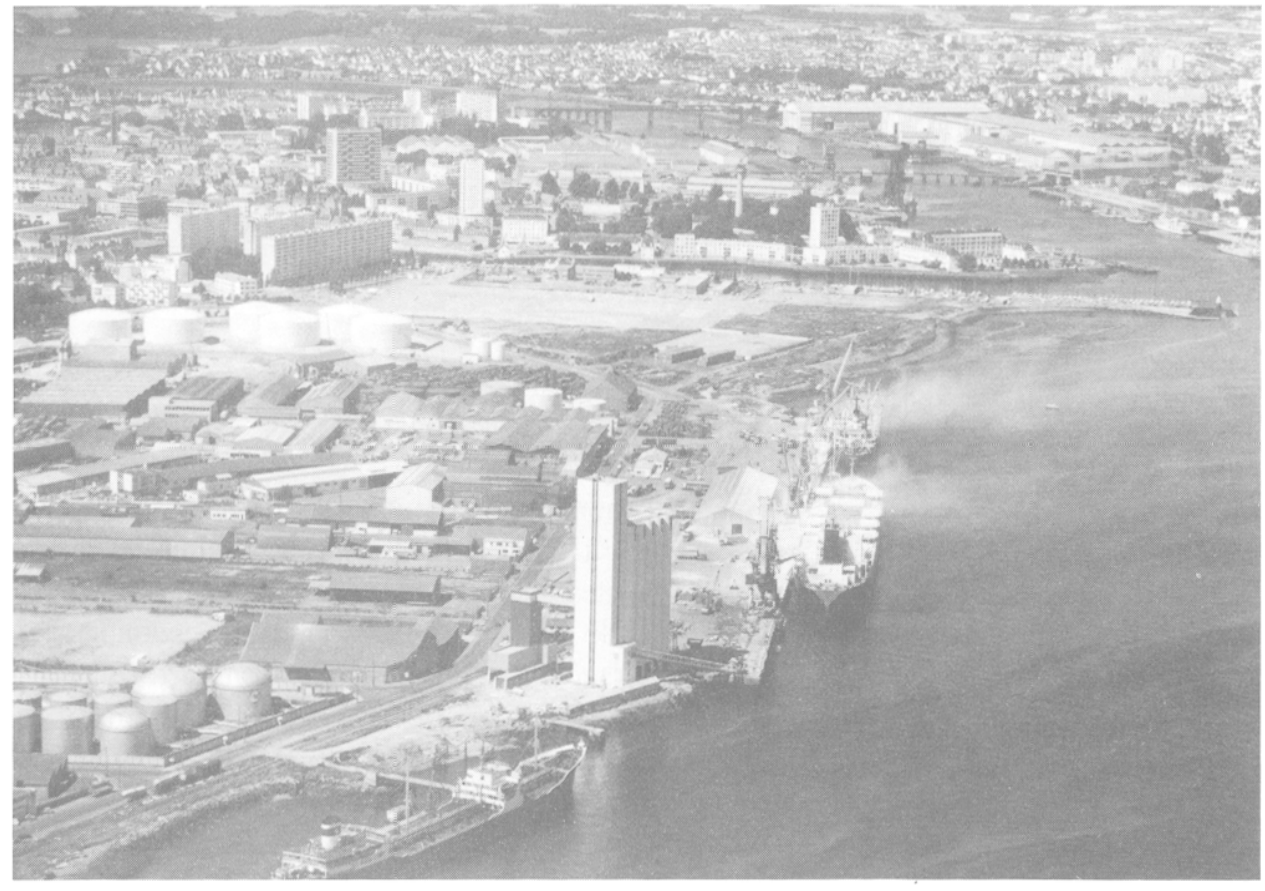

Lorient, arsenal et port de commerce. gnent au plus près les activités universitaires et/ou économiques (CNRS, INSERM, INRA) et les organismes beaucoup plus finalisés, de dimension plus réduite. mobilisant des équipements très spécifiques dans un nombre d'implantations restreint. Ainsi, près de la moitié des effectifs de l'IFREMER («qui est naturellement décentralisé du fait de ses objectifs, de ses missions et de sa proximité nécessaire avec les milieux professionnels, principalement dans le domaine des ressources vivantes»22) travaillent en Bretagne. Ces organismes et la stratégie de rééquilibrage des activités de recherche sur le territoire national (politique de délocalisation entreprise par le CNRS) ne peuvent faire plus qu'atténuer les phénomènes de polarisation au profit de l'Ilede-France avec le risque ${ }^{23}$ de renforcer la concentration de la recherche amont à Paris, la province étant davantage vouée à l'application.

Pour l'enseignement, polarisation et hiérarchisation sont à la fois quantitatives et qualitatives. Si l'offre universitaire de Brest s'est étoffée d'année en année, celle de Toulon reste incomplète et sélective : au côté des trois cycles en sciences économiques et droit. les formations scientifiques ne sont représentées que par le DEUG A et ses prolongements en second et troisième cycles; les formations littéraires sont encore plus minces avec le seul DEUG LEA. En fait, le nombre et la diversité des diplômes préparés sont en relation étroite avec la taille et demande sociale (formations banales). Reflets de contrastes socio-économiques et de déterminismes locaux (les sciences de la mer à Brest, la défense à Toulon), les résidus ne réduisent pas vraiment les disparités hiérarchiques inter-urbaines.

Au total, la création de nouveaux sites universitaires et les tentatives de diffusion de la recherche ne réduisent pas les écarts entre centre et périphérie et pourraient au contraire affermir le pouvoir de commandement de Paris et des grandes métropoles régionales ${ }^{24}$. Or, par la jeunesse de leur histoire universitaire, les ports militaires font encore figure de périphérie dans le tissu universitaire français.

20. Curien $H_{\text {., }}$ "La localisation de la recherche», petit-déjeuner de presse du Ministre de la Recherche et de la Technologie, 30 janvier 1992.

21. Brunet R., "L'université, la ville et la région», L'Espace géographique 1991.3, p. 212.

22. Mailfert A., Recherche et ferritoire, La Documentation française 1991, p. 36.

23. Risque déjà observé pour l'INRA d'après Beckouche $P$., "Une région qui commande la recherche nationale mais la développe peu», Annales de la recherche urbaine 1991, N 50 , pp. 30-37.

24. L'observation a été faite pour le Québec par Bruneau P., Les villes moyennes au Québec. Leur place duns le système socio-spatial, Presses de l'Université du Québec, 1989 : "A une périphérie spécialisée, voire ultra-spécialisée, s'oppose un centre tout à la fois polyvalent, diversifiè et spécialisé». 


\section{La banalisation des universités de proximité?}

La spécialisation et l'accent mis sur les formations technologiques et professionnalisées sont efficaces en termes de rentabilité médiatique puisque seules les «petites nouvelles», en l'occurrence Le Havre, Pau, Chambéry, qui «proposent des formations très professionnalisées en liaison avec l'économie locale» semblent, en termes de représentations et de satisfaction étudiantes, capables de rivaliser avec les plus prestigieuses ${ }^{25}$.

Cette spécialisation suppose une forte mobilité d'étudiants adoptant un comportement de consommateurs et s'inscrivant dans la logique d'un marché de l'enseignement supérieur où le choix de la formation suivie serait largement fonction des perspectives ultérieures de classement social. Une grande attention serait alors portée au lieu de consommation en fonction des représentations que les jeunes et leurs parents se font des différents types de formations et des différentes universités. Cette attitude est surtout celle des meilleurs élèves de terminale en faveur des formations sélectives (IUT, CPGE) avec des mouvements ultérieurs de retour à l'université aux

\begin{tabular}{|c|c|c|c|c|}
\hline \multicolumn{5}{|c|}{$\begin{array}{l}\text { Les premiers cycles dans les universités bretonnes } \\
\text { (en \% des effectifs de la filière dans l'université) }\end{array}$} \\
\hline $\begin{array}{l}\text { Université } \\
\text { Lettres } \\
\text { Droit Eco AES } \\
\text { Sciences } \\
\text { Med } \\
\text { Pharma Dent }\end{array}$ & $\begin{array}{c}\text { Rennes1 } \\
58.5 \\
45.9 \\
\\
30.6\end{array}$ & $\begin{array}{c}\text { Rennes2 } \\
49.3 \\
65.9\end{array}$ & $\begin{array}{l}\text { UBO } \\
65.7 \\
71.9 \\
66.5\end{array}$ & $\begin{array}{c}\text { Bretagne } \\
52.9 \\
63.8 \\
52.1\end{array}$ \\
\hline Total & 46.8 & 51.1 & 64.2 & 52.8 \\
\hline
\end{tabular}

Source : Rapport Morvan d'après Rectorat

NB : Les effectifs de Lettres à Rennes 1 (200 étudiants de philosophie) et de sciences à Rennes 2 ( 81 étudiants de MASS) n'ont pas été pris en compte dans les calculs par université.

niveaux des deuxième et troisième cycles, tendant ainsi à «constituer le premier cycle comme zone de repli ou d'attente pour des bacheliers médiocres ${ }^{26}$.

Dans les sites universitaires récents, les effectifs de premier cycle sont relativement plus importants qu'ailleurs parce que le manque de durée n'a pas permis le développement d'un grand nombre de formations de recherche et d'enseignements de troisième cycle (cf. tableau). Ces universités peuvent difficilement échapper à la contradiction entre les ambitieux désirs de distinction universitaire et les contraintes imposées par la demande sociale, la volonté politique d'élévation du niveau moyen de qualification scolaire des nouvelles générations d'actifs, les limites quantitatives des solutions alternatives (IUT, STS) qui poussent vers l'université des cohortes de plus

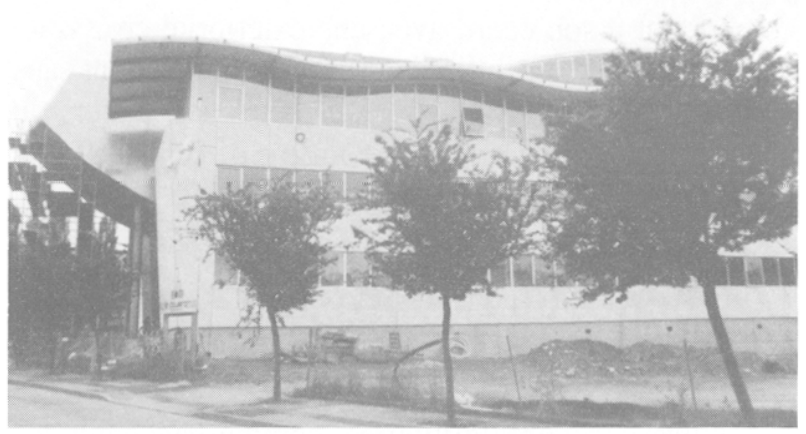

Lorient. Juillet 92. Nouveau bâtiment universitarre Lanveur.

en plus nombreuses et diversifiées dans leurs origines sociales, culturelles et scolaires. La majorité choisira des formations générales à gros effectifs, notamment dans le secteur des langues et des sciences humaines et sociales. Avec l'élargissement de sa base sociale, l'université s'est banalisée au point de devenir un service de moyenne proximité. A cette évolution correspond une logique de dispersion et de diffusion spatiale : «Chaque fois que le statut d'un service a évolué en ce sens, les centres dispensateurs du service se sont multipliés, et la portée géographique moyenne du service a diminué. Cette diffusion résulte des intérêts convergents des usagers et des promoteurs du service, ici la puissance publique. Les premiers cherchent à minimiser les coûts du déplacement nécessaire pour accéder au service, les seconds se satisfont d'une moindre portée géographique du marché quand, dans une population donnée, l'intensité de la demande augmente ${ }^{27}$. Cette organisation en fonction des bassins de clientèle régionale potentielle entre en contradiction avec la logique de polarisation et de spécialisation liée à la fonction recherche et à la professionnalisation des enseignements. D'où le risque de remise en cause de la traditionnelle liaison structurelle entre enseignement et recherche et de secondarisation des nouveaux sites universitaires.

Sous l'effet de cette diffusion, les recrutements sont de plus départementalisés, ce qui contredit l'hypothèse de la mobilité associée à la spécialisation, surtout au niveau des premiers cycles où les étudiants sont de plus en plus nombreux à vivre comme des lycéens prolongés chez leurs parents (cas de $56 \%$ des étudiants lorientais). Le nombre d'étudiants augmente donc par la production d'étudiants qui ne le sont pas au sens culturel du mot.

25. «Universités : le palmarès des étudiants》, Le Monde de l'éducation, juillet-août 1992.

26. Berthelot J.M., "Les effets pervers de l'expansion des enseignements supérieurs : le cas de la France.", Sociétés contemporaines 1990.4, p. 115.

27. Saint-Julien T., "L'université et l'aménagement du territoire», L'espace géographique, 1990-1991, 3, p. 206. 
Même si l'université reste porteuse d'un espoir de promotion sociale pour ses nouveaux venus, elle a perdu beaucoup de sa dimension symbolique. Les étudiants se comportent à son égard avec une extériorité croissante jusqu'à adopter une attitude d'usagers qui passent sans s'investir. Peuvent-ils dans ce cas être des acteurs du changement d'image et du dynamisme de ces villes sans réelle histoire universitaire?

\section{Les limites du renouveau par l'université}

Les villes portuaires militaires, comme beaucoup des villes accédant à la fonction universitaire ou en phase de développement universitaire, sont tiraillées entre, d'une part, la spécialisation dictée par l'économie et la rentabilité en terme d'image externe et, d'autre part, la polyvalence et les formations banales imposées par le souci de la régulation sociale et par le surpeuplement des mêmes formations dans les pôles universitaires régionaux. Guidées par l'idée de relations créatrices d'emplois entre universités et entreprises et par le projet d'embauche locale des diplômés, ces villes veulent des formations et des recherches en synergie avec l'économie locale. Les projets locaux pour l'université et la recherche prennent corps au sein de l'hypothèse technopolitaine de synergie entre recherche, enseignement supérieur et entreprises de haute technologie. Ces aspirations sont en contradiction avec les intérêts des métropoles régionales, habituées à tirer les profits dc la polarisation. Par ailleurs, ces projets souvent ambitieux risquent de ne concerner que des effectifs modestes en regard de la croissance actuelle de la demande de formations supérieures, dans des disciplines valorisantes mais sensibles aux effets de mode.

Elles étaient prêtes à des efforts financiers importants à condition que ce soit pour ces formations porteuses de modernité el de technicité, avec des deuxième et troisième cycles aussi variés que possible, et avec des infrastructures de recherche attractives, dans l'espoir d'une redynamisation et d'un changement d'image. Devant la perspective de n'être que des universités périphériques, quand elles font la part des choses entre les retombées possibles et le mythe et quand elles mesurent les limites de leurs capacités à retenir localement une jeunesse de plus en plus formée, elles deviennent comptables de leurs investissements.... L'exemple des réticences de l'UBO pour ouvrir un Deug de psychologie afin de résorber les sureffectifs de Rennes 2 montre les limites de l'effort consenti pour le développement des filières de culture générale. Aujourd'hui, nous savons que l'Institut Européen des Sciences de la Mer ne sera pas construit sur le port de Brest ; le navire lorientais, sans être véritablement en position périphérique, a été construit dans le quartier de Lanveur où existaient des opportunités foncières, mais situé à quinze minutes de marche du centre-ville. Comment, alors que les discours sur l'Université semblent davantage relever de l'incantatoire que de la réelle volonté, demander aux étudiants, en supposant qu'ils soient assez nombreux pour cela, d'animer les centres-villes, et aux universitaires d'être les promoteurs d'un regain de dynamisme pour des villes en crise identitaire?

Raymonde Séchet

Raymonde Séchet. membre de I" URA 915 CNRS. enseigne la géographie sociale à l' Université de Rennes 2. Flle participe activement aux programmes de recherche sur les relations Ville-Université lancés par le Ministère de l'Education Nationale et le Plan Urbain. tout en poursuivant des travaux sur les villes militaires portuaires d' Europe occidentale. 